
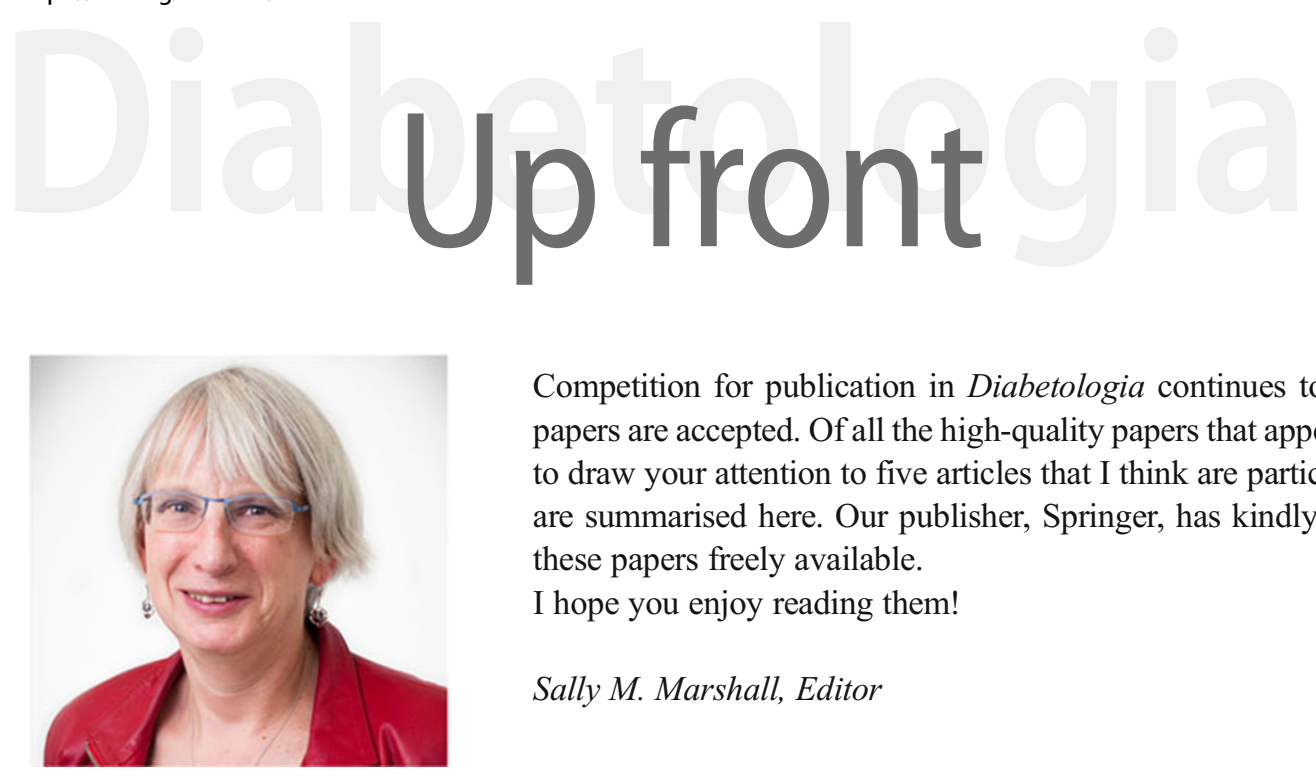

Competition for publication in Diabetologia continues to grow, and less than $20 \%$ of papers are accepted. Of all the high-quality papers that appear in this month's issue I want to draw your attention to five articles that I think are particularly interesting. The articles are summarised here. Our publisher, Springer, has kindly made the full text of each of these papers freely available.

I hope you enjoy reading them!

Sally M. Marshall, Editor

\section{Chrono-nutrition for the prevention and treatment of obesity and type 2 diabetes: from mice to men}

John A. Hawley, Paolo Sassone-Corsi, Juleen R. Zierath

In the current 'obesogenic' environment there is growing popular and scientific interest in how the timing and frequency of meals impacts metabolic health, with an appreciation that the duration of which food is consumed effects numerous physiological and metabolic processes. Such 'chrono-nutrition' is aimed at optimising metabolism by timing nutrient intake to the acrophases of daily metabolic rhythms via manipulation of the feeding-fasting cycle. In this issue, Hawley and colleagues (https://doi.org/10.1007/s00125-02005238-w) review recent research from both animal and human studies demonstrating that dietary protocols incorporating chronic energy restriction, intermittent fasting and timerestricted feeding improve multiple cardiometabolic markers associated with obesity and type 2 diabetes risk. The figure in this review is available as a downloadable slide.

Next steps in the identification of gene targets for type 1 diabetes

Struan F. A. Grant, Andrew D. Wells, Stephen S. Rich

Type 1 diabetes is characterised by young age at onset, requirement for exogenous insulin for survival and a strong genetic predisposition in the absence of family history. Genetics account for approximately half of total risk, yet, unlike other diseases, a single genomic region (HLA) constitutes $\sim 50 \%$ of that genetic component. Much of the remaining genetic risk has been identified, with the vast majority of variants residing in DNA regulatory regions. In this issue, Grant et al (https://doi.org/10.1007/s00125-020-05248-8) provide a perspective on the transition from an allelic association to the effect of genetic variation on tissue-specific expression, identification of target genes of the associated variants and use of genetic data to identify those individuals at risk of type 1 diabetes prior to clinical presence of symptoms. The authors suggest that functional genomic studies, increased ethnic diversity of individuals included in such studies, and improved characterisation of chromosomal landscapes will be necessary to reveal novel prevention and treatment strategies. The figure in this review is available as a downloadable slide.

Association of early-onset diabetes, prediabetes and early glycaemic recovery with the risk of all-cause and cardiovascular mortality

Sung Min Kim, Gyeongsil Lee, Seulggie Choi, Kyuwoong Kim, Su-Min Jeong, Joung Sik Son, Jae-Moon Yun, Sin Gon Kim, Seung-sik Hwang, Seong Yong Park, Yeon-Yong Kim, Sang Min Park

Recently, the increasing incidence of diabetes among young adults aged 20-30 years has added to the overall disease burden. Individuals with early-onset diabetes have similar or higher cardiovascular risk than those with late-onset diabetes. 
In this issue, Kim and Lee and colleagues (https://doi.org/10. 1007/s00125-020-05252-y) investigated whether newly diagnosed early-onset diabetes, prediabetes and glycaemic recovery among young adults is associated with incident cardiovascular disease or mortality using data from a nationwide cohort study of 2,502,375 young adults without cardiovascular disease at baseline. They report that individuals with early-onset diabetes and prediabetes had increased risks of incident CVD and all-cause mortality at the 10 year follow up. Notably, in individuals with early glycaemic recovery from diabetes or prediabetes, the risks of incident 10-year CVD and all-cause or CVD mortality were reduced. The authors conclude that maintenance of glycaemic control in young adults may be important for the prevention of CVD and all-cause mortality.

An atlas on risk factors for type 2 diabetes: a wide-angled Mendelian randomisation study

Shuai Yuan, Susanna C. Larsson

Observational studies have revealed a large number of possible risk factors for type 2 diabetes but whether the reported associations are causal remains unclear. In this issue, Shuai Yuan and Susanna Larsson (https://doi.org/10.1007/s00125020-05253-x) identified causal associations of 34 exposures (18 risk factors and 16 protective factors) with type 2 diabetes under a wide-angled Mendelian randomisation framework. Insomnia was identified as a novel causal risk factor for type 2 diabetes. Eight associations remained after adjustment for adulthood BMI. In addition, there were 21 possible associations. The authors conclude that these findings imply that

All text supplied by the authors. prevention strategies for type 2 diabetes need to be considered from multiple perspectives.

Increase in endogenous glucose production with SGLT2 inhibition is attenuated in individuals who underwent kidney transplantation and bilateral native nephrectomy

Giuseppe Daniele, Carolina Solis-Herrera, Angela Dardano, Andrea Mari, Andrea Tura, Laura Giusti, Jancy J. Kurumthodathu, Beatrice Campi, Alessandro Saba, Anna Maria Bianchi, Carla Tregnaghi, Maria Francesca Egidi, Muhammad Abdul-Ghani, Ralph DeFronzo, Stefano Del Prato

The expected reduction in plasma glucose concentration mediated by sodium-glucose cotransporter 2 (SGLT2) inhibitors is partially offset by a concomitant increase in endogenous glucose production. The signal mediating such a paradoxical response to SLT2-inhibition is currently unknown. In this issue, Daniele, Solis-Herrera et al (https://doi.org/10. 1007/s00125-020-05254-w) demonstrate that SGLT2 inhibition in non-diabetic individuals who have undergone kidney transplantation with removal of native kidneys is associated with attenuation of the paradoxical stimulation of endogenous glucose production. They explain that these results suggest the existence of a neuronal axis linking the kidney and the liver that modulates endogenous glucose production in response to urinary glucose loss induced by SGLT2 inhibition. Based on these findings, the authors propose that SGLT2 inhibition can activate efferent renal nerves, generating a signal that augments endogenous (hepatic) glucose production to compensate for the urinary glucose loss. 